\title{
Diacronie
}

Studi di Storia Contemporanea

$\mathrm{N}^{\circ} 24,4 \mid 2015$

Le dittature militari: fisionomia ed eredità politica

\section{Marion Fontaine, Fin d'un monde ouvrier. Liévin, 1974}

\section{Francesca Sanna}

\section{(2) OpenEdition}

\section{Journals}

Edizione digitale

URL: http://journals.openedition.org/diacronie/3603

DOI: $10.4000 /$ diacronie.3603

ISSN: 2038-0925

\section{Editore}

Association culturelle Diacronie

Notizia bibliografica digitale

Francesca Sanna, « Marion Fontaine, Fin d'un monde ouvrier. Liévin, 1974 », Diacronie [Online], N²4, 4 l

2015, documento 22, Messo online il 29 décembre 2015, consultato il 24 septembre 2020. URL :

http://journals.openedition.org/diacronie/3603 ; DOI : https://doi.org/10.4000/diacronie.3603 


\section{Diacronie}

N. 24 | 4|2015 Le dittature militari: fisionomia ed eredità politica

$\mathbf{2 2 / ~}$

\section{RECENSIONE:}

\section{Marion FONTAINE, Fin d'un monde ouvrier. Liévin, 1974, Paris, Éditions de l'EHESS, 2014, 240 pp.}

a cura di Francesca SANNA *

Marion Fontaine - maître de conférences presso l'Università di Avignone e ricercatrice al Centre Norbert Elias (UMR 8562) - è autrice di numerosi saggi e contributi relativi al mondo operaio delle miniere francesi del secondo dopoguerra. In special modo, si è occupata dei movimenti politici e sindacali fra i minatori del Nord et Pas de Calais con un peculiare focus sull'uso politico dello sport. Fra le sue pubblicazioni più note: Le Racing Club de Lens et les «Gueles Noires» e la codirezione di Une contre-histoire de la III République ${ }^{2}$.

Il saggio Fin d'un monde ouvrier. Liévin, 1974 si inserisce perfettamente fra i temi di suo maggior interesse. A partire dall'analisi di un disastro minerario, la catastrofe di Liévin appunto, l'autrice delinea i tratti della più importante regione carbonifera della Francia negli anni Sessanta e Settanta, intersecando le questioni politiche, sociali ed economiche emerse nel dopo-catastrofe.

Alle 6 del mattino del 27 dicembre 1974, nel giorno della riapertura della miniera dopo le vacanze natalizie, nella Fosse 3 bis dell'Unité de Production 19 di Liévin, un'esplosione uccise sul colpo i 41 minatori discesi al lavoro poche ore prima, mentre un altro operaio morì il giorno successivo per i traumi riportati.

La tragedia produsse uno shock tale da muovere il governo nazionale, che il giorno stesso inviò sul luogo il Ministro dell'Industria, Michel d'Ornano, e, in seguito, il Primo Ministro, Jacques Chirac, a presenziare i funerali delle vittime il 31 dicembre.

${ }^{1}$ FONTAINE, Marion, Le Racing Club de Lens et les « Gueules Noires ». Essai d'histoire sociale, Paris, Les Indes Savantes, 2010.

${ }^{2}$ FONTAINE, Marion, MONIER, Frédéric, PROCHASSON, Christophe, Une contre-histoire de la IIIe République, Paris, La Découverte, 2013. 
Subito ebbero inizio le indagini, che si concentrarono inizialmente sulla natura dell'incidente: fu un coup de grisou o un coup de poussières? La questione, al di là delle specifiche tecniche, portava davanti agli occhi della Francia non solo un mondo industriale considerato ormai un dinosauro della modernità, ma rinfocolava anche le polemiche sulla questione delle responsabilità e della sicurezza del lavoro. Indagini e processi durarono quasi dieci anni, mentre le speculazioni della stampa, dopo l'emozione iniziale, si ridussero alle pagine della sola cronaca locale.

Gli eventi che seguirono la tragedia e i motivi di questa «implosione» di interesse sono i temi sondati da Marion Fontaine nel primo capitolo del suo libro, il cui titolo Un anachronisme? Une catastrophe minière en 1974 già anticipa una prima tesi sul portato dell'evento. $\mathrm{Al}$ di là della rievocazione dei fantasmi di antiche catastrofi - quella di Courrière ad esempio - Marion Fontaine interpreta lo scivolamento nazionale nell'oblio come un sintomo visibile della percezione della deindustrializzazione a cui il sistema carbonifero francese andava incontro negli anni Settanta. La scomparsa degli aggiornamenti dalle testate nazionali, eccetto forse quelle più vicine alla classe operaia come «Libération», sarebbe stata dunque una diretta conseguenza della graduale e inconscia assunzione che quel mondo stava morendo e che l'attenzione dell'opinione pubblica era ormai proiettata altrove.

Non si era più nell'epoca dei Trente Glorieuses, si era, infatti, al termine della decadenza, in procinto di attraversare il guado verso nuovi assetti economici con la dismissione dei settori della grande produzione in serie e delle antiche fonti energetiche, quella carbonifera in primis.

I protagonisti della passata stagione industriale sarebbero perciò spariti con essa, 0 no? La catastrofe di Liévin sembrava concretizzare tragicamente questo atto di morte e non pochi si esercitarono nel suo necrologio.

Il comportamento della stampa, fra il lutto e l'oblio, nella sua volontà di rappresentare a tinte fosche il mondo minerario, ebbe gioco facile nel riesumare l'immagine zoliana del minatore: quel misero eroe che, dalle pagine di Gérminal, emerge afflitto da una sorte decisa dalla miniera. Eroe, nell'affrontare ogni giorno la minaccia della morte sotterranea, è tuttavia eroe misero, senza speranza: da quel mondo non vi è scampo né con l'azione politica, né con l'atto violento, ma solo con l'estremo atto, la morte.

3 Il Coup de grisou è un'esplosione provocata dal contatto fra l'aria e il gas grisou, tipico delle miniere di carbone, mentre il coup de poussière è un'esplosione provocata dall'incendio delle polveri di carbone in sospensione. URL: < http://www.infomine.com/dictionary/ > [consultato il 4 aprile 2015]. 
I toni con cui si descrisse la tragedia risalgono ancora a quell'immaginario, arricchito però dalla sovrapposizione successiva dell'eroe militante, raffigurazione costruita fra gli anni della Resistenza e quelli della successiva Battaglia del Carbone. Il minatore allora era anche eroe patriottico, circondato quasi naturalmente da un'aura comunista. Questa figura mitica non rimase però a solo appannaggio della stampa, ma venne subito assorbita dalle formazioni politiche di sinistra che militavano nell'area di Liévin, primo fra tutti il Partito comunista rivoluzionario marxista leninista (PCR-lm). Ma era un'immagine davvero rappresentativa?

All'epoca della catastrofe di Liévin si era già alla metà degli anni Settanta e Marion Fontaine si chiede appunto, fra la seconda e la terza parte del saggio, se questo discorso simbolico potesse avere ancora un rimarchevole valore politico. La risposta, come si può immaginare, è no. L'immagine del minatore come eroe oppresso, patriottico e militante non poteva più fungere, al momento dell'incidente di Liévin, nel 1974, come un dispositivo di «accensione della rivoluzione», poiché il milieu di quei simboli stava scomparendo e i minatori stavano abbandonando loro stessi quei miti, insieme alla speranza che il loro mondo restasse com'era. E allora perché le formazioni politiche e sociali presenti sul luogo continuarono ad usarli? Cecità? Incapacità di analisi? O una semplice resistenza al cambiamento?

In questo comportamento, secondo Fontaine, non solo si intrecciano i seppur flebili echi del '68 che ancora animavano i respiri dei militanti di estrema sinistra, ma anche la questione della lotta alle Houillères ${ }^{4}$, sia per ostacolare i loro piani di rapida dismissione delle coltivazioni, sia per stabilirne più in generale le responsabilità nella sicurezza sul lavoro, particolarmente per gli incidenti e le malattie professionali.

$\grave{E}$ in questo quadro che entra in gioco la scena processuale come arena di scontro fra queste fazioni. L'istruttoria ufficiale fu affidata al giudice Henri Pascal, procuratore di Béthune, già famoso nell'ambiente per aver condotto il processo sullo scabroso affaire di Fouquières et Bruay5.

\footnotetext{
${ }^{4}$ Le Houillères du bassin du Nord et Pas de Calais (HBNPC) dipendenti da Charbonnages de France era il gruppo preposto alla gestione dei giacimenti nella regione francese del Nord et Pas de Calais dopo la nazionalizzazione delle miniere avvenuta al termine della Seconda Guerra Mondiale.

5 L'affaire di Fouquières et Bruay, nella primavera del 1972, fu l'ambiguo processo per l'omicidio di Brigitte Dewèvre, figlia di minatori, in cui venne condannato e poi assolto un notaio, Pierre Leroy. I processo, gestito appunto da Henri Pascal, prese subito i contorni di un teatro politico sfruttato dai militanti del PCR-lm, in cui la povera figlia del proletariato era stata circuita e poi uccisa dall'infido figlio della borghesia. Nonostante il notaio fosse stato scagionato dalla confessione, poi ritrattata, di un amico della giovane, l'impatto simbolico di quel processo rimase a lungo nella memoria del territorio. Il ponte fra questo caso e la catastrofe di Liévin è testimoniato dal fatto che i parenti di Brigitte parteciparono al tribunale popolare di Liévin, accostando i minatori uccisi alla loro figlia e il notaio alle Houillères, definendo i tratti di una
} 
Parallelamente al processo ufficiale vi fu anche l'iniziativa di un gruppo eterogeneo di militanti legati al PCR-lm e battezzata «tribunale popolare di Liévin». Tuttavia, nonostante il fatto che persino Jean Paul Sartre avesse partecipato, dichiarando che l'incidente in miniera non era mai un evento casuale, il tribunale popolare non ebbe grande effetto a causa, soprattutto, del poco radicamento degli organizzatori sul territorio.

Ciò si riaggancia alla questione culturale precedente. I militanti gauchistes volevano riesumare un mito passato per riaccendere la lotta presente, ma ne ignoravano l'obsolescenza nella funzione di sprone rivoluzionario, senza contare la confusione ideologica che creavano cercando di usare un vecchio simbolo del PCF.

Secondo Fontaine, infatti, il problema di fondo della questione risiedeva nel fatto che i militanti di sinistra non si rendevano conto delle trasformazioni in atto e quindi non erano in grado di trarre conclusioni utili alla riproduzione della loro causa ed infine di se stessi ${ }^{6}$.

Il vero cuore del «tema processuale» è, perciò, la vicenda del giudice Pascal e del processo ufficiale. Qui, ogni elemento concorre a tracciare un uso del diritto in senso politico, all'interno di un'arena quasi teatrale costituita dall'aula del tribunale. Attorno a questo palcoscenico Marion Fontaine fa ruotare attori ed eventi, delineando appunto quel complicato quadro di intersezioni fra le questioni economica, sociale, politica e culturale del mondo carbonifero in rotta.

Si può dire, inoltre, che il processo per la catastrofe di Liévin ebbe i tratti di un affaire, poiché la sua celebrazione non spiccò tanto per la ricerca di una verità evenemenziale, quanto soprattutto per la messa a nudo di dinamiche di impresa ritenute illegali e disumane. L'apparato giudiziario mise in scena uno scontro frontale fra, da un lato, i rappresentanti delle Houillères e, dall'altro, sindacati, partiti e l'intera comunità mineraria, nel frattempo e non senza difficoltà aggregatasi in diversi gruppi e associazioni. Il processo di Liévin, però, non si può classificare come un affaire classico, come quello interpretato da Liora Israël nel suo testo Le armi del diritto ${ }^{7}$, poiché non furono i militanti o i loro simpatizzanti i veri attori del processo, ma il giudice Pascal che, per sua stessa ammissione, non agiva nelle logiche di uno schieramento politico, ma secondo una sua propria visione del dispositivo giudiziario. La sua azione fu purtuttavia politica nel senso che travalicò il suo fine diretto, quello di scoprire la verità

sovrapposizione dicotomica vittima-carnefice politicizzata dall'identificazione nella lotta di classe proletari versus borghesi.

${ }^{6}$ FONTAINE, Marion, Fin d'un monde ouvrier. Liévin, 1974, Paris, Éditions de l'EHESS, 2014, p. 40 .

7 ISRAËL, Liora, Le armi del diritto, Milano, Giuffré, 2012. 
sul disastro minerario, per assumere i caratteri di un'accusa al sistema e di un’ingiunzione di cambiamento, oltre che di risarcimento. Secondo Marion Fontaine, l'impegno di Henri Pascal in questo processo non fu quello di un magistrato tradizionale, ma quello di un «porte-voix d'une volonté de justice ouvrière» incrociando, senza però condividere, le aspirazioni della sinistra proletaria ${ }^{8}$.

Il giudice Pascal non era del resto estraneo a questo genere di situazioni dato che già nell'ambito dell'affaire di Fouquières et Bruay i militanti di sinistra avevano cercato di trasformare il fatto di cronaca in un palcoscenico di lotta di classe. Il fatto che lo stesso giudice si occupasse anche del caso di Liévin aveva certamente influito sull'immaginario dell'opinione pubblica, creando un ponte neppure troppo implicito fra i due eventi.

Ma un processo alle Houillères, come istituzione, era fattibile? Questo è ciò che si domanda Fontaine. C'erano stati altri casi prima di Liévin - nello specifico Courrière nel 1906 e Marcinelles nel 1956 i più eclatanti -, ma il sistema giudiziario non era stato in grado di andare oltre i propri strumenti, mentre il giudice Pascal intendeva farlo, incorporando nell'accusa il tema dell'ipersfruttamento economico, campo dell'etica del lavoro. Per fare ciò non solo si distaccò dagli esperti incaricati degli studi sulle dinamiche specifiche dell'incidente, ma soprattutto si rivolse costantemente alla stampa, creando un caso mediatico.

Giunsero dunque a contrapporsi due visioni della tragedia: una, quella delle Houillères, che si concentrava sulle perizie tecniche e sul Règlement general des Mines per dimostrare l'accidentalità dell'evento, e un'altra, quella del giudice Pascal, fondata sul principio morale della responsabilità per la morte dei lavoratori, causata della negligenza dell'impresa.

In questa dinamica, Pascal riuscì a mettere a nudo non solo l'esasperato particolarismo delle Houillères, ma anche la loro politica di disimpegno, in primis finanziario, che rendeva ogni giorno più pericoloso lavorare in miniera.

Il risultato di questo scontro fu la messa sotto accusa dell'ingegnere capo dell'UP 19 Augustin Coquidé, il quale entrò nel processo come il simbolo di quella responsabilità che gli uni negavano di avere e gli altri affermavano di poter pretendere. Tuttavia, il caso Coquidé finì per essere l'ultimo atto di Pascal nel processo di Liévin poiché, dopo il ricorso delle Houillères, la Chambre d'accusation di Douai sollevò il giudice dall'incarico a causa di alcune scorrettezze procedurali.

Il processo, tuttavia, non si fermò, ma si trasformò in interminabili requisitorie basate solamente sulle perizie dei tecnici: la catastrofe si ridusse, dunque, alla sua 
dimensione visibile, quantificabile e contingente. Venne meno quella visione di insieme che rendeva il processo un affaire, mettendo le Houillères al sicuro da accuse che ne avrebbero colpito l'istituzione nel suo complesso.

Il confronto con un caso di morte sul lavoro che avvenne nello stesso periodo a Vendin-le-Vieil, non lontano da Lens, non fece che enfatizzare il progressivo svilimento del portato morale del processo di Liévin9. L'affaire Chapron, come venne denominato, fu, infatti, davvero condotto con armi politiche e il giudice, Patrice de Charette, si rivelò juge rouge per antonomasia. Egli incontrò, senza discostarsene, le formazioni politiche e sindacali di sinistra per un'azione collettiva nei conflitti legati ai rapporti di lavoro. Il caso Chapron è perciò molto più vicino al concetto di diritto come arma politica e non a caso Marion Fontaine cita proprio qui Liora Israël e il suo testo, già ricordato più sopra.

Ritornando però agli anni del processo, si comprende chiaramente che nelle forze sociali e nei protagonisti politici continuò a regnare la confusione e l'incapacità di capire cosa stava avvenendo nel bacino del Nord et Pas de Calais. Ci si interrogava vanamente sulla ragione per cui il popolo di Liévin non avesse apparentemente intentato alcuna azione per riaffermare i propri diritti violati e si rispondeva a tale domanda con una semplificazione, che accusava gli stessi lavoratori di apatia.

In realtà, un accenno di movimento sociale è testimoniato dall'azione della CFDT (Confédération française démocratique du travail) e dalla creazione dell'Associazione di difesa degli interessi morali e materiali delle famiglie delle vittime della catastrofe. Se quest'ultima perorava la sua causa non più con la lotta rivoluzionaria, ma con l'uso dei media, il secondo, distinguendosi dai sindacati tradizionali, riuscì a comprendere le trasformazioni in atto nell'ambiente minerario e a denunciare la vera composizione di cause della catastrofe di Liévin attraverso un ritorno al diritto come arma politica.

È particolarmente eloquente il fatto che la CFDT, sia per il caso di Liévin sia per il disastro di Merlebach in Lorena, si sia valso della collaborazione di Heri Leclerc, famoso avvocato militante fin dal 1968.

Nonostante questa agitazione politica, il processo si concluse il 23 gennaio $1981 \mathrm{con}$ un verdetto insignificante: le Houillères erano ritenute civilmente responsabili e l'ing. Coquidé fu condannato a pagare un'ammenda. Nessuna parola venne spesa sulla responsabilità dell'impresa nella sicurezza sul lavoro. Secondo l'autrice, vi fu alla base un problema di scarto temporale. Il processo avvenne troppo presto rispetto al cammino legislativo in materia e troppo tardi rispetto al cambiamento economico: il

9 Il 29 settembre 1975, in seguito a un incidente nella fabbrica HGD (Huiles et Goudrons dérivés) che costò la morte di un lavoratore interinale, il giudice Patrice de Charette accusò il direttore della fabbrica Jean Chapron di omicidio involontario con carcerazione preventiva. MARION, Fontaine, op. cit., p. 149. 
settore minerario, come si è detto in precedenza, era ormai considerato vestigia del passato, non provocava una partecipazione dell'opinione pubblica. Liévin può però essere ritenuto a buon diritto un cippo sulla strada del riconoscimento di quella responsabilità, che avverrà, per la Francia, con i verdetti della Cassazione sull'amianto nel 2002.

Emerge qui un ponte fra gli ultimi interventi di Marion Fontaine in materia di storia mineraria a proposito appunto degli incidenti e delle malattie professionali. Quale fil rouge lega i disastri visibili delle distruzioni a quelli invisibili delle malattie degenerative come la silicosi? Entrambi sono il prodotto del lavoro, compiuto in condizioni di sicurezza non rispettate. Come dimostra il caso Liévin, fino a non molti decenni fa, non si riteneva l'impresa responsabile delle conseguenze che il lavoro infliggeva agli operai e perciò non la si imputava colpevole. Nel caso della miniera si evocava la fatalità, come a dire che accettare quel lavoro significava accettarne anche imprevedibili eventualità. Non molto tempo fa, di fronte ad una vera e propria strage di minatori causata da un incidente, un noto capo di stato si espresse in termini non molto dissimili da questa visione del lavoro svolto in miniera ${ }^{10}$.

Potrebbe anche sembrare a prima vista che gli incidenti sul lavoro, non solo nell'ambito minerario, siano causati dagli stessi lavoratori per una negligenza sulle norme di sicurezza, ma questa ricostruzione è appunto un'apparenza. Marion Fontaine lo mostra a chiare lettere sia nel testo su Liévin sia nel suo saggio sulle malattie professionali raccolto in Santé et Travail à la mine. XIXème XXème siècle ${ }^{11}$. Sia gli incidenti che le malattie professionali sono il prodotto delle medesime condizioni, create non dal lavoratore ma dall'impresa: l'obsolescenza degli strumenti di sicurezza e l'organizzazione del lavoro. Pochi investimenti e negligenza nel rinnovo delle attrezzature non possono essere imputabili al lavoratore, né gli si può rimproverare la scarsità di pause di riposo o lo stress da superlavoro. Alla base dell'errore umano che porta il lavoratore a far del male a se stesso e agli altri non vi è una deliberata volontà, ma l'accumularsi di fattori sfavorevoli che aumentano la percentuale di potenziali incidenti. In questo consiste la responsabilità dell'impresa, cioè nell'evitare questa accumulazione di fattori sfavorevoli.

${ }^{10} \mathrm{Ci}$ si riferisce a quanto dichiarato dal presidente Turco Erdoğan in occasione della tragedia mineraria di Soma del Maggio 2014 e riportato dai giornali di tutto il mondo. «Les accidents sont dans la nature même des mines» «Turquie : le bilan définitif de la catastrophe minière s'élève à 301 morts», in Le Monde, 17 Maggio 2014,

URL: < http://www.lemonde.fr/europe/article/2014/o5/17/turquie-le-bilan-de-la-catastropheminiere-porte-a-299-morts_4420480_3214.html > [consultato il 4 aprile 2015]

${ }^{11}$ MARION, Fontaine, Silicose et extrême gauche dans le Nord dans les années 7o. Les raisons d'une aporie, in RAINHORN, Judith (dir.), Santé et Travail à la mine. XIXème XXème siècle, Villeneuve-d'Ascq, Presses Universitaires du Septentrion, 2014. 
Nell'ultimo capitolo del saggio, Marion Fontaine riporta lo sguardo agli anni presenti, dimostrando che il suo studio non è la ricostruzione di un ambiente defunto, ma un vero problema storico. La regione del Nord et Pas de Calais vive continuamente a contatto col suo passato, sia fisicamente, sia nell'immaginario, perciò la studiosa si chiede che posto occupi la catastrofe di Liévin in questo pantheon di figure e di valori. Gli abitanti di Liévin sembrano non aver mai fatto pace con la loro tragedia, cercano di dimenticarsene nascondendone le vestigia, come la polvere sotto i tappeti, ma ad uno sguardo più attento questo comportamento è un segno di disagio. Fra i vari indizi riportati da Marion Fontaine, il più visibile è forse quello dei monumenti commemorativi. Uno fra tutti pare piuttosto eloquente: la visione di una scultura, raffigurante il crollo della galleria che sommerge i minatori, posta in mezzo a una rotonda all'entrata di un centro commerciale ${ }^{12}$.

Al termine di questi sei capitoli, la conclusione ripercorre le tre impressioni della studiosa sulla sua materia di analisi: il pessimismo, l'interesse e le domande. Tutte e tre sono ampiamente condivisibili. Se il pessimismo emerge dagli amari risultati di una prassi giudiziaria che si ripete frequentemente in casi di responsabilità penale delle imprese per la sicurezza dei lavoratori, nondimeno rimane vivo l’interesse nel comprenderne i significati, dato che ogni evento rinvia non solo all'ambiente in cui si è prodotto, ma anche a una comparazione fra storie simili. Ed, infine, le domande, sorte dal tentativo di comprensione degli eventi e del loro contesto, generano sempre riflessioni sul presente, a cui sono legate le persistenze e le rimozioni della storia dalla memoria e dal paesaggio. Nel caso di Liévin e del Nord et Pas de Calais, anche se il mito della miniera è finito, non per questo mancano i progetti per valorizzare l'identità scaturita da quel lavoro. Ne sono un esempio ben visibile e di successo le esperienze del Centre Historique Minier du Nord et Pas de Calais e, ancor di più, del Louvre di Lens, che ha dedicato tanta parte del suo spazio espositivo al mondo in cui è stato collocato. Queste esperienze confermano il pensiero della studiosa che afferma, nelle battute finali del suo libro che: «Le fait de non plus croire aux mythes n'implique pas forcément la fin des projets intellectuels ou politiques ${ }^{13}$.

${ }^{12}$ Ibidem, p. 201.

13 Ibidem, p. 227. 


\section{* L'autore}

Francesca Sanna ha conseguito la Laura Triennale in Storia con lode presso l'Università degli studi di Milano nel 2012 con una tesi sul profilo storico-economico dell'industria estrattiva sarda del secondo dopoguerra. Attualmente è iscritta alla Laurea Magistrale in Scienze storiche dell'Università di Bologna ed è stata selezionata per partecipare al Corso integrato Storia e civiltà comparate in cotutela con l’Université Denis Diderot - Paris 7.

URL: < http://www.studistorici.com/progett/autori/\#Sanna_Francesca >

\section{Per citare questo articolo:}

SANNA, Francesca, «Recensione: Marion FONTAINE, Fin d'un monde ouvrier. Liévin, 1974, Paris, Éditions de I'EHESS, 2014, 240 pp.», Diacronie. Studi di Storia Contemporanea : Le dittature militari: fisionomia ed eredità politica, 29/12/2015,

URL:<http://www.studistorici.com/2015/12/29/sanna_numero_24/ >

Diacronie Studi di Storia Contemporanea $\}$ www.diacronie.it

Risorsa digitale indipendente a carattere storiografico. Uscita trimestrale.

redazione.diacronie@hotmail.it

Comitato di redazione: Jacopo Bassi - Luca Bufarale - Elisa Grandi - Antonio César Moreno Cantano - Deborah Paci - Fausto Pietrancosta - Alessandro Salvador - Matteo Tomasoni - Luca Zuccolo

Diritti: gli articoli di Diacronie. Studi di Storia Contemporanea sono pubblicati sotto licenza Creative Commons 3.0. Possono essere riprodotti e modificati a patto di indicare eventuali modifiche dei contenuti, di riconoscere la paternità dell'opera e di condividerla allo stesso modo. La citazione di estratti è comunque sempre autorizzata, nei limiti previsti dalla legge. 